\title{
Teaching Benchmark Strategy for Fifth-Graders in Taiwan
}

\author{
Der-Ching Yang ${ }^{1} \&$ M. L. Lai ${ }^{2}$ \\ ${ }^{1}$ Graduate Institute of Mathematics and Science Education, National Chiayi University, Chiayi, Taiwan \\ ${ }^{2}$ Early Childhood Education, National Chiayi University, Chiayi, Taiwan \\ Correspondence: M. L. Lai, Early Childhood Education, National Chiayi University, Chiayi, Taiwan. Tel: \\ 886-5-226-3411 Ext 1551. E-mail: mlai1@mail.ncyu.edu.tw
}

Received: December 14, 2012

Accepted: February 24, 2013

Online Published: April 2, 2013

doi:10.5539/jel.v2n2p69

URL: http://dx.doi.org/10.5539/jel.v2n2p69

\begin{abstract}
The key purpose of this study was how we taught the use of benchmark strategy when comparing fraction for fifth-graders in Taiwan. 26 fifth graders from a public elementary in south Taiwan were selected to join this study. Results of this case study showed that students had a much progress on the use of benchmark strategy when comparing fraction after the experiment. This indicated that an experienced teacher played a key role and knew how to help their children lean the use of benchmark appropriately when comparing fraction.
\end{abstract}

Keyword: benchmak, fifth-grader, comparing fraction

\section{Introduction}

Fractions play an important role in the mathematics curriculum, but the learning of fractions is often the most difficult part for children (Cramer, Post, \& delMas, 2002; National Council of Teachers of Mathematics [NCTM], 2000; National Mathematics Advisory Panel, 2008; Yang, Reys, \& Wu, 2010). The earlier studies (Empson, 2003; Yang, \& Reys, 2002) showed that many students often hold some misconceptions on fractions. Most students suffer when learning fractions: one $5^{\text {th }}$ grader in our study said, "I am not good at fractions because I can only do addition and subtraction of fractions."; while another commented, "I am always confused with fractions." As a result of this, many teachers have difficulties teaching fractions, as indicated by one participating teacher, "It is so hard to teach fractions!" Previous studies (Cramer et al., 2002; Reys \& Yang, 1998; Yang, 2005; Yang, Li, \& Lin, 2008) found while they can mechanically compute fractions (e.g., $\frac{3}{5}>\frac{4}{9}$ ) using the common denominator strategy, children often cannot justify their answer. The benchmark strategy (i.e., $\frac{1}{2}$ is considered a key benchmark when comparing $\frac{3}{5}$ and $\frac{4}{9}$ because the former is larger and the latter is less than $\frac{1}{2}$ ); however, might empower children to develop conceptual understanding on fraction (Crammer et al.). To solve this problem, this article reports how the benchmark strategy was introduced at a $5^{\text {th }}$ grade class in Taiwan to help children develop a better fractional sense. In fact, this case study show that how teacher translating big ideas into practice to support the requirement for implementation of Common Core State Standards for Mathematics [CCSSM] (Phillips \& Wong, 2012).

\section{Background}

The ability to use benchmarks to solve numerical problems has been considered to be a key characteristic of number sense (NCTM, 2000; Reys \& Yang, 1998; Yang, 2005; Verschaffel, et al., 2007; Yang \& Wu, 2010). However, the findings of several studies showed that it is difficult for the elementary and middle grades students to develop and use a benchmark to solve arithmetic problems (Cramer et al., 2002; Markovits \& Sowder, 1994; 
Reys \& Yang, 1998; Yang, 2005). For example, the study of Reys \& Yang (1998) found that there were nearly two-thirds of 6th-grade students in Taiwan correctly computed the complex written computation $\frac{12}{13}+\frac{7}{8}$; however, over three-quarters of these students failed to use benchmark (e.g., use 1 as a benchmark to decide $\frac{12}{13}$ is near 1 and $\frac{7}{8}$ is also near 1) to estimate the answer as being close to the number 2. Obviously, these students do not make sense what they are doing or what $\frac{12}{13}$ and $\frac{7}{8}$ really mean. While these students mechanically applied the written method they had learned in school mathematics class, their efforts did not reflect number sense. This situation happens frequently in traditional mathematics teaching. Our pilot study in a fifth grade class in Taiwan also found the similar problem. The finding is described as foillows:

\subsection{Misconceptions on Comparing Fractions}

To begin with, children were asked to sort the fractions $\frac{1}{2}, \frac{4}{9}$, and $\frac{3}{5}$ from the largest to the smallest. Among these $5^{\text {th }}$ graders, 7 out of 26 respondents correctly answered this problem by adopting the common denominator strategy though they could not justify their answer. Three children could not solve this question at all. The other children's responses were categorized into four types as follows:

1) 5 out of 26 respondents believed that the smaller the denominator is, the larger the fraction is, and thus $\frac{1}{2}>\frac{3}{5}>\frac{4}{9}$. One of them even "proved" his idea using the following example: $\frac{1}{4}>\frac{2}{9}>\frac{3}{17}$, because $4<9<17$.

These participants only considered the sizes of denominators when comparing fractions, which implies that they did not truly understand the fraction.

2) 3 out of 26 repondents believed that $\frac{1}{2}>\frac{4}{9}>\frac{3}{5}$, and showed this by using the pictorial representation in the context of the continuous quantity, such as with a pizza (see figure 1). The following example showed that these students lacked the important concept of unit (same unit) when comparing fractions.
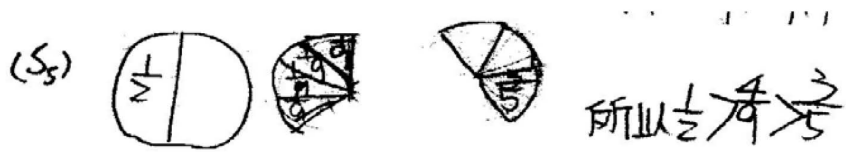

Figure 1. Children's drawings to campare fractions using the continuous quantity

3) 5 out of 26 respondents represented the problem using discrete quantities as the figure 2 below shows, and believed that $\frac{1}{2}<\frac{3}{5}<\frac{4}{9}$. Obviously, they simply decided the magnitude of these fractions by comparing the sizes of the numerators. 


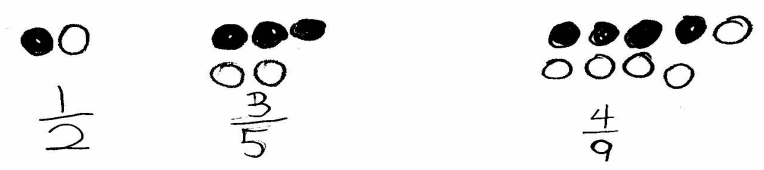

Figure 2. Children's drawings to campare fractions using the discrete quantity

Apparently, these students' understanding of the fraction was influenced by the whole number concept and they did not define the role of unit before comparing fractions with different denominators.

4) 3 out of 26 respondents answered $\frac{4}{9}>\frac{3}{5}>\frac{1}{2}$ by simply comparing the magnitude of numerators and denominators because $4>3>1$ and $9>5>2$. These students treated fractions as whole numbers and thus responded based on their knowledge of whole numbers.

Based on the findings above, obviously, a majority of participants had misconceptions of fractions because of an incomplete understanding of fraction. Surprisingly, none of the students applied $\frac{1}{2}$ as a "benchmark" to solve the problem.

In response to children's poor performance on comparing fractions, the benchmark strategy was explicitly taught and the following strategies were designed to enhance children's understanding of the fraction in general and to foster their competence of flexibly comparing fractions in specific.

\section{Results}

\subsection{Enhancing Children's Understanding of the Fraction by Adopting Pictorial Representations}

Cramer et al. (2002) suggested that children's mental image of the fraction concept, specifically the concept of unit, could be reinforced by pictorial representation. To begin, the teacher encourages students to draw pictures representing $\frac{4}{9}$ on the blackboard (see Figure 3, each work is numbered), followed by a teacher-student discussion.

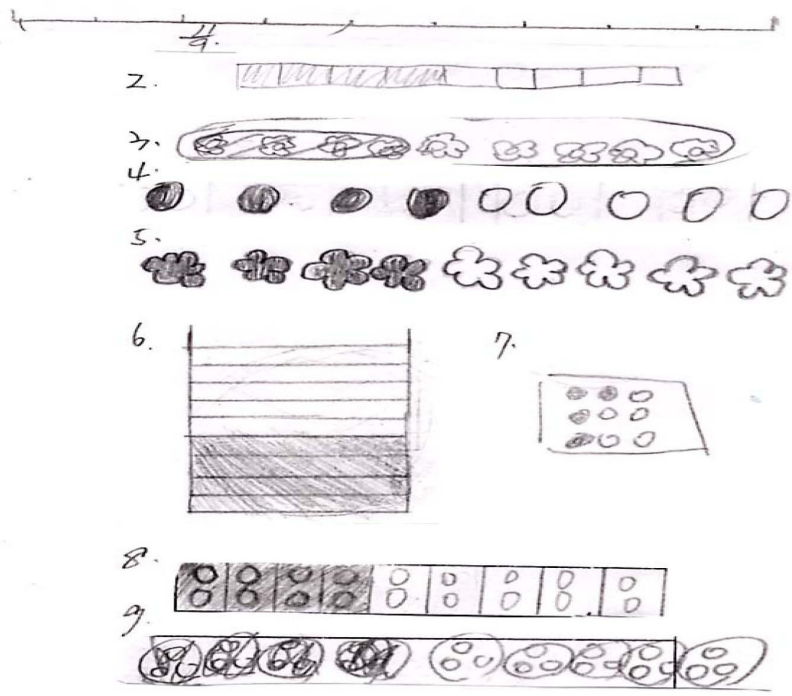

Figure 3. Students' drawings of 4/9 
T: Very good, you came up with several ways of representation the fraction. Do you think all of the examples of $\frac{4}{9}$ on the blackboard are correct?

S: Yes, they are all correct!

T: Does anyone have any questions?

S7: Why are there two circles in each piece? (Pointing at the eighth figure)

S4: Here are eighteen, and if they are divided equally by nine, each cell will have two.

T: Can you understand? (S7 says "Yes".) Then, would you please explain ninth figure?

S7: Here are 27. They are divided by nine equally, and four pieces equals twelve.

T: How many are there in each piece?

S7: Three.

T: Well, Please tell me: if I want four in each piece, how many are there in total?

S7: (Thinks for a while.) Thirty-six.

Through discussion with students, it is obvious that they made great progress in understanding fractions. For example, fractions can be represented in various ways depending on the unit used in each situation; as graphs 7 , 8 and 9 show in Figure 4, we can see that they are drawn using the discrete quantity method. By means of the drawing experience, students get to understand the importance of the units - if the numbers of units aquong fractions are different, the number in each piece will be different though they are all the representations of $\frac{4}{9}$.

Through the discussion of the pictorial representations such as drawings, 3 5 in Figure 4, the students appeared to have a clear concept about what the unit quantity means in the fraction. The teacher then moved on to the next stage and asked students to compare fractions using pictorial representations.

\subsection{Leading Children to Compare Fractions Using Pictorial Representations}

The teacher then asked the students to think about how to represent the fraction $\frac{3}{5}$ in a similar way as the previous fraction, $\frac{4}{9}$.

T: Now, how can we compare $\frac{3}{5}$ and $\frac{4}{9}$ ?

$\mathrm{S}:$ We could use the common denominator strategy and simply compare the numerators.

T: Can you tell me why? [suddenly, the students become speechless]

The common denominator strategy is certainly a correct procedure, yet most students do not understand the rationale of this solution. We hoped that the students could think from the concept of fractions. This strategy might be able to confirm and clarify their concepts.

T: Besides using the common denominator strategy, are there other solutions? For example, can we compare them as Figure 3 above shows?

S19: There are 3 shaded circles in $\frac{3}{5}$ and 4 shaded circles in $\frac{4}{9}$, thus $\frac{4}{9}>\frac{3}{5}$.

S6: No, because the denominators on both fractions are not the same.

$\mathrm{T}$ : Why can we not compare them when the denominators are not the same?

S6: Because it is not fair.

T: Okay. So, what condition do we have to take into account when comparing fractions?

$\mathrm{S}$ : They should have the same unit quantity, i.e., the same denominator.

T: Great! How about this one? (see Figure1)

$\mathrm{S}$ : It is not correct either, because they are not the same size. 
The discussions above showed that students understood the importance of unit quantity and they knew that the unit quantity must be the same when comparing fractions. In order to make sure that they could apply the pictorial representations to compare $\frac{4}{9}$ and $\frac{3}{5}$, the teacher continued and extended the discussions as below:

T: Can anyone tell me $\frac{4}{9}$ or $\frac{3}{5}$ is larger by using pictorial representations?

S12: As the Figure 4 shows, $\frac{3}{5}$ is larger because the indicated length is longer.

S (some students show their pictures): Yes!(Examples of students'solution are shown below).

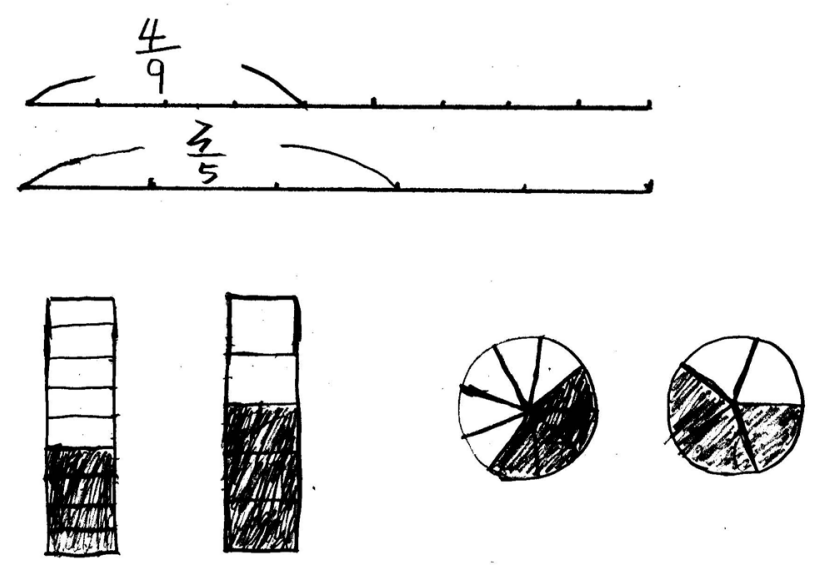

Figure 4. Students' drawings of $\frac{4}{9}$ and $\frac{3}{5}$

From the responses of the students, the observations and queries, the teacher confirmed that most of her students understood how to compare fractions using pictorial representations. She thought it is a good time to introduce the idea of the benchmark strategy (e.g., using $\frac{1}{2}$ as the benchmark), which is listed next.

\subsection{Trying to Apply Initial Symbolic Representations from Pictorial Representations}

The teacher asked students whether or not $\frac{3}{5}$ or $\frac{4}{9}$ was greater than $\frac{1}{2}$. The purpose was to guide students into using symbolic rather than pictorial representation. Students seemed to be confused about this problem. Thus, the teacher asked the students to draw a picture of $\frac{1}{2}$ as a reference. Then, three students were inspired by the strategy and became able to respond to teacher's question. The discussions were observed as follows:

S5: Check if the numerator is greater than half of the denominator.

S3: Draw a line in your mind and then mark where the fraction is on the line.

S2: You can double the numerator and see whether or not the number is greater than the denominator, or divide the denominator by two to compare it with the numerator.

The solutions of the three students above showed that they truly understood the question posed by their teacher. Unfortunately, it seemed very difficult for most of the students. 


\subsection{Connecting Pictorial Representations to Symbolic Representations by Oral Communication}

To better develop the students' understanding and to improve student' abilities to represent fractions symbolically, the teacher invited S5 to explain how she applied $\frac{1}{2}$ as the benchmark to her classmates. Theoretically, children can use their own language to help others understand abstract concepts:

S5: The numerator of $\frac{3}{5}$ is greater than half of the denominator, so the fraction is greater than $\frac{1}{2}$; the numerator of $\frac{4}{9}$ is less than half of the denominator, so the fraction is less than $\frac{1}{2}$.

S5's explanations imply that she can use the symbolic representation, which impressed the teacher. However, many students were still confused so the teacher continued:

T: Some students do not seem to understand this. Can someone help me explain this in more detail or does anyone have any different solutions?

S3: First, imagine the figure of a fraction in your mind and see if the numerator is greater than half of the denominator.

$\mathrm{T}$ : How can we imagine a figure of the fraction where the numerator is greater than half of the denominator?

S3: That means when you draw the figure to represent $\frac{3}{5}$, the parts to represent the numerator are greater than half of the denominator. However, in the figure of $\frac{4}{9}$, its numerator is less than half of the denominator 9.

T: Very good! Now you can think and compare your thoughts with what S3 said. That is, when you imagine the drawing of $\frac{3}{5}$ in your mind, the parts to represent the numerator are greater than half of the denominator. However, when drawing a figure to represent $\frac{4}{9}$, the parts to represent the numerator are less than half of the denominator. As S5 said, the numerator of $\frac{3}{5}$ is greater than half of the denominator, so the fraction is greater than $\frac{1}{2}$. Besides, since the numerator of $\frac{4}{9}$ is less than half of the denominator, that fraction is less than $\frac{1}{2}$. Do you agree?

S: Yes! $\frac{3}{5}$ is greater than $\frac{1}{2} ; \frac{4}{9}$ is less than $\frac{1}{2}$.

Many students understood S3's explanations, which is probably because S3's explanations transformed the pictorial representation to a psychological operation. Although many students had not yet applied S5's explanation, they made good progress in understanding and applying symbolic representations if they accepted and used S3's explanation.

\subsection{Post-Instruction Evaluation}

To evaluate the benefit of benchmark strategy, children were asked to determine whether $\frac{5}{8}$ or $\frac{2}{5}$ is larger, and why? Surprisingly, except for two students, 24 out of 26 students successfully solved it. Among them, eleven adopted the pictorial representations shown below (see Figure 5). 


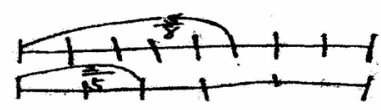

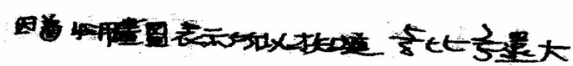
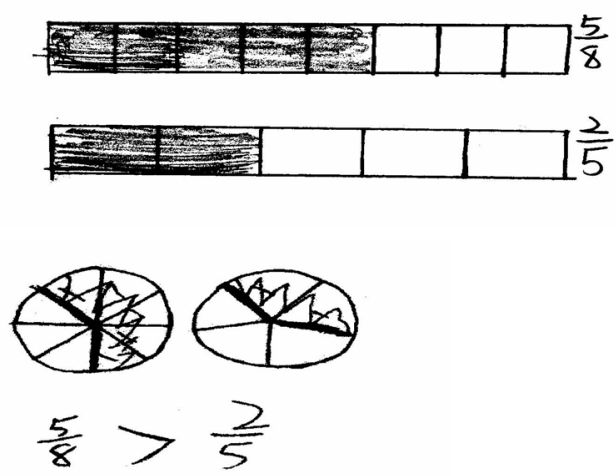

Figure 5. Students' drawings of $\frac{5}{8}$ and $\frac{2}{5}$

Surprisingly, 13 out of 26 students adopted the benchmark heuristics shown below (see Figure 6) to solve this problem. Compared with their performance before the introduction of the benchmark strategy, the difference was statistically significant $(p<.001$, Sign Test).
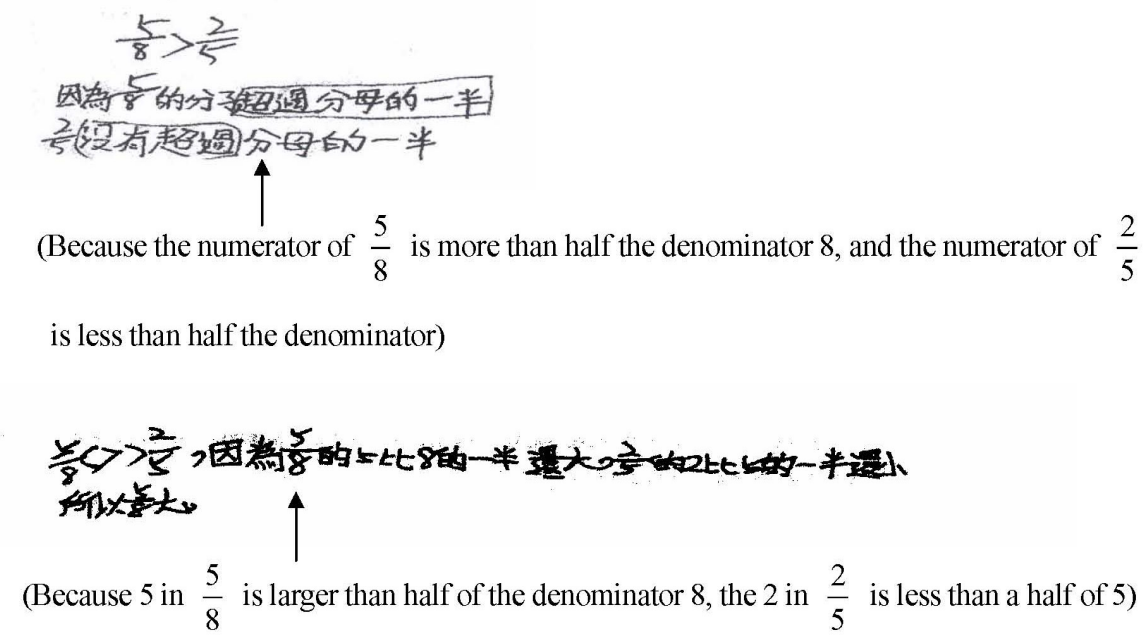

Figure 6. Students' solutions using the benchmark heuristics

The teacher continued by asking an advanced question, described below, to further examine whether or not students could compare fractions mentally without using pictorial representations or paper-and-pencil methods. Which one, $\frac{5}{11}$ or $\frac{4}{7}$, is larger and why? 
S (many students answered): $\frac{4}{7}$ is larger.

T: Why? (most of the students raised their hands). S3 please tell us your reasons.

S3: $\frac{4}{7}$ is larger, because $\frac{4}{7}>\frac{1}{2}$ and $\frac{1}{2}>\frac{5}{11}$.

T: Why $\frac{4}{7}$ is greater than $\frac{1}{2}$ and $\frac{1}{2}$ is greater than $\frac{5}{11}$ ?

S12: Because 4 is more than a half of 7 and 5 is less than a half of 11 .

From the students' responses, it was obvious that the students did understand better how to compare fractions, even though some of them still had difficulties using the benchmark strategy. Under the guidance of the teacher and communicating with their classmates, our participants had a chance to learn how to use pictorial representations to enhance their understanding and to translate their pictorial representations into symbolic ones. It seems reasonable to believe that under the guidance and help of more capable people, children can learn better.

\section{Discussion and Conclusion}

The findings derived from the study are threefold:

First, the benchmark strategy greatly enhances children's understanding of fraction. The major finding of this study is that most children, 24 out of 26 , are able to adopt the benchmark strategy successfully carrying out fraction comparison and thus children's understanding of fraction is greatly improved via this explicitly-taught heuristic. The mathematics textbooks used in Taiwan and Singapore unfortunately do not include this useful heuristic. Therefore, what most children could do to compare fraction probably simply adopting the energy-consuming and effortful common denominator strategy, which greatly limits their conceptual understanding on fractions (Reys \& Yang, 1998). Overall, this study suggests that the benchmark strategy should be taught explicitly and by doing so, not only can children make sense fractions but also flexibly and efficiently solve problem.

Seoncd, flexible use of multiple representations is a good way to develop a better understanding of mathematical concepts. The process of bridging the road from pictorial to symbolic representations, as was evident in this study, indicates that students can develop a better understanding of fractions and, more specifically, of the use of benchmark strategies. Flexible use of multiple representations is an important process in learning mathematics. The findings of this study show that students' understanding of the fraction became more robust and solid by means of such multiple representations. This is consistent with the findings of earlier study (Cramer, et al., 2002) that profound understanding of mathematical concepts and high-level mathematical thinking relies on the flexible use of multiple representations.

Third, teacher-children interactions cultivate children's positive disposition. Through discussions and communication, children have a better understanding and learn how to share their opinions with others. Teachers should respect what children think, accept their mistakes, and inspire them with learning opportunities. Under the atmosphere of interaction and cooperation, it produces some learning momentum that enlightens students and initiates their motivation. Through the discussions and conversations, students can clarify their thinking. These results are consistent with the suggestion of earlier studies (Empson, 2003; Yang, 2006) that if teachers can encourage students timely, have more interactions among students and teachers, and then those students who have lower achievements can develop their mathematical ability also.

Children may make various mistakes and may have misconceptions when they learn about fractions. This is because students do not properly understand the meaning of fractions. When comparing fractions, for example, many students do not have clear concepts about fractions and ignore the role of unit quantity. From this case study, it is obvious that the most important goal for a teacher is to check if the students understand, and to know whether or not they have any misconceptions. We also realized that a teacher should be on the same side as his or her students, and should listen to what they think. If teachers do not pay sttention to students' responses, they usually think that their teaching is effective. Therefore, we should try our best to let students express their thoughts in class, and to try to solve misconceptions as they arise. The findings of this study support the statement of Phillips \& Wong (2012) that teachers should "focus and spend more time on fewer, more important things so students can build conceptual understanding, achieve procedural skill and fluency, and learn how to 
transfer what they know to solve problems in and out of the math classrooms (p. 31).

\section{Educational Implications}

The educational implications are described as follows:

First, children can develop and use the benchmark flexibly under appropriate teaching approach and leading. This supports the idea that providing children with more learning opportunities and multiple learning environments promotes the development of number sense.

Second, Children can learn better through appropriate learning environments which encourage students to discuss, collaborate, share, and clarify their ideas.

\section{Acknowledgment}

The authors thank all the reviewers and Editor for their comments. This paper is part of a research project supported by the NSC, Taiwan with grant no. NSC 100-2511-S-415 -008 -MY3. Any opinions expressed here are those of the authors and do not necessarily reflect the views of the NSC, Taiwan.

\section{References}

Cramer, K. A., Post, T. R., \& delMas, R. C. (2002). Initial fraction learning by fourth-and fifth-grade students: A comparison of the effects of using commercial curricula with the effects of using the rational number project curriculum. Journal for Research in Mathematics Education, 33(2), 111-144. http://dx.doi.org/10.2307/749646

Empson, S. B. (2003). Low-Performing Students and Teaching Fraction for Understanding: An Interactional Analysis. Journal for Research in Mathematics Education, 34(4), 305-343. http://dx.doi.org/10.2307/30034786

Markovits, Z., \& Sowder, J. T. (1994). Developing number sense: An intervention study in grade 7. Journal for Research in Mathematics Education, 25(1), 4-29. http://dx.doi.org/10.2307/749290

National Council of Teachers of Mathematics. (2000). Principles and Standards for School Mathematics. Reston, VA: Author.

National Mathematics Advisory Panel. (2008). Foundations for success: The final report of the national mathematics advisory panel. Washington, DC: U.S. Department of Education.

Phillips, V., \& Wong, C. (2012). Teaching to the Common Core by design, not accident. Phi Delta Kappan, 93(7). 31-37.

Reys, R. E., \& Yang, D. C. (1998). Relationship between computational performance and number sense among sixth- and eighth-grade students in Taiwan. Journal for Research in Mathematics Education, 29(2), 225-237. http://dx.doi.org/10.2307/749900

Yang, D. C. (2005). Number sense strategies used by sixth grade students in Taiwan. Educational Studies, 31(3), 317-334. http://dx.doi.org/10.1080/03055690500236845

Yang, D. C. (2006). Developing number sense through real-life situations in school of Taiwan, Teaching Children Mathematics, 13(2), 104-110.

Yang, D. C., Li, M. N., \& Lin, C. I. (2008). A study of the performance of 5th graders in number sense and its relationship to achievement in mathematics. International Journal of Science and Mathematics Education, 6(4), 789-807. http://dx.doi.org/10.1007/s10763-007-9100-0

Yang, D. C., \& Reys, R. E. (2002). Fractional Number Sense Strategies Possessed by Sixth Grade Students in Taiwan. Hiroshima Journal of Mathematics Education, 10(December), 53-70.

Yang, D. C., Reys, R. E., \& Wu, L. L. (2010). Comparing how fractions were developed in textbooks used by the $5^{\text {th }}$ - and $6^{\text {th }}$-graders in Singapore, Taiwan, and the U.S.A. School Science and Mathematics, 110(3), 118-127. http://dx.doi.org/10.1111/j.1949-8594.2010.00015.x 